\title{
Warum sich qualifizierte Migranten nicht bei der Polizei bewerben.
}

Andreas Majewski

\section{Einleitung}

Ende Oktober letzten Jahres wurde dem 50-jährigen Jubiläum des Anwerbeabkommens zwischen Deutschland und der Türkei gedacht. ${ }^{1}$ Wirtschaftliche Erfolgsgeschichten wie die deutscher Automobilkonzerne wären ohne die Arbeitskraft von Menschen aus Italien und der Türkei nicht möglich gewesen.

Auch die Polizeibehörden versuchen seit mehr als zwanzig Jahren überwiegend erfolglos, den Anteil von Polizeibeamten mit Migrationshintergrund $\mathrm{zu}$ steigern. ${ }^{2}$ Die maßgeblichen Folgen der demografischen Entwicklung, also die Alterung der Bevölkerung bei gleichzeitiger Abnahme der Gesamtbevölkerungszahl (Statistisches Bundesamt 2009, S. 5 - 6) und der überproportionale Anstieg des Anteils von Menschen mit Migrationshintergrund $(\mathrm{MH})$ an der Gesamtbevölkerung, insbesondere in der Gruppe der unter 21-Jährigen (Die Beauftragte der Bundesregierung für Migration, Flüchtlinge und Integration 2010, S. 40 41), erhöhen den Handlungsdruck auf Verantwortungsträger in den Polizeibehörden.

$\mathrm{Zu}$ den Vorteilen und Notwendigkeiten einerseits und Hindernissen sowie Widerständen andererseits ist viel Forschung betrieben worden (Behr 2007; Blom 2005; Dittmer 2008; Drechsler und Nagora 2007; Dudek 2009; Hunold 2008).

In der vorgestellten Studie steht dagegen die subjektive Seite, die Sicht von möglichen Berufsanfängern, also von Abiturienten mit $\mathrm{MH}$, die in Berlin potentielle Bewerber für die Polizeilaufbahn des gehobenen Dienstes darstellen, im Vordergrund. ${ }^{3}$ Den Erfahrungen der Einstellungsabteilung der Berliner Polizei zufolge gibt es gemessen am Anteil an der entsprechenden Altersgruppe relativ wenige Bewerber und Bewerberinnen von Menschen mit Migrationshintergrund. Viel dürften wegen des Numerus Clausus' von 3,0 gar nicht erst zum Auswahlverfahren zugelassen werden oder am Auswahlverfahren scheitern (Drechsler und Nagora 2007, S. 27 f. bezogen auf die Jahre 20062010). Dies erscheint nicht überraschend, da ein Migrationshintergrund auf allen
Stufen des Bildungssystems zu Benachteiligungen führt und Migranten vergleichsweise weniger oft das Abitur ablegen (Autorengruppe Bildungsberichterstattung 2010, S. 205 - 206). Dagegen ist die Quote derer, die die Oberschule beenden und ein Studium beginnen, bei Studienberechtigten mit Migrationshintergrund sogar signifikant höher als bei Deutschstämmigen (ebd. S. 119, 289).

Es bewerben sich also offenbar die „Falschen “, die am Einstellungstest scheitern, während sich die „Richtigen“ anderen Hochschulen zuwenden. Dabei ist die Polizei kein unattraktiver Arbeitgeber, da sie in Zeiten prekärer Arbeits- und Lebensverhältnisse ein verbindliches Arbeitsverhältnis und somit einen materiellen Anreiz bereits während des Studiums offeriert. ${ }^{4}$

Was also hindert leistungsstarke Schulabsolventen mit MH daran, sich bei der Polizei zu bewerben?

\section{Der Ist-Zustand}

Statistische Zahlen zu dem Anteil von Polizeibeamten mit $\mathrm{MH}$ in den deutschen Polizeibehörden liegen nicht vor, da sie aus rechtlichen Erwägungen nicht erhoben werden. In Berlin wird der Anteil auf 1,5\% geschätzt (Hunold 2008, S. 31 - Stand 2006), obwohl die Einstellung von ausländischen Bürgern in die Polizei dort seit 1979 öffentlich diskutiert (durch den damaligen Innensenator Ulrich) und seit 1988 in der Praxis ermöglicht wurde (durch den damaligen Innensenator Kewenig). Man kann die bisherigen Bemühungen, mehr Migranten einzustellen, also als gescheitert bezeichnen (Jaschke 2006; zit. n. Behr 2007, S. 292).

\section{Die Rahmenbedingungen}

\subsection{Verschärfung der Problematik} durch den demografische Wandel

Die Bevölkerungsabnahme hat u. a. einen Rückgang der Anzahl von Schulabsolventen zur Folge. Dieser wird zu einem größeren Wettbewerb um qualifizierte Bewerber bzw. Arbeitnehmer führen, der sich bereits jetzt beispielsweise beim Bedarf an Lehrern oder im Bereich der Metallberufe abzeichnet (Autorengruppe Bildungsberichterstattung 2010, S. 76, 103).

Der hohe Anteil von Migranten bei den unter 6-Jährigen bei gleichzeitigen Rückgang der Zahl von Kindern ohne $\mathrm{MH}$ wird zum Ergebnis haben, dass die Polizei jedenfalls in Großstädten wie Berlin in spätestens zehn bzw. zwölf Jahren, also wenn die jetzt unter 6-Jährigen beginnen, die Schulen zu verlassen, über ein bereits bewährtes zielgruppenorientiertes Personalrekrutierungsprogramm verfügen muss, um einerseits den eigenen Personalbedarf überhaupt decken zu können, anderseits den gesellschaftlichen Realitäten ansatzweise gerecht zu werden ${ }^{5}$.

\subsection{Bildungsnachteile durch Migrationshintergrund}

Ein Zuwanderungshintergrund führt zu einer divergierende Bildungsbeteiligung, die bereits beim Besuch von Kindertagesstätten mit einer deutlich geringeren Beteiligungsquote von Kindern mit $\mathrm{MH}^{6}$ beginnt (Autorengruppe Bildungsberichterstattung 2010, S. 52). Die Ungleichheit setzt sich fort, da Kinder mit $\mathrm{MH}$ beim Übergang in die Schule überproportional von Rückstellungen als Ergebnis der Schuleingangsuntersuchung betroffen sind (ebd., S. 59). Die festgestellten Disparitäten bestätigen sich schließlich bei den Schulabschlüssen: Migranten besitzen infolge der schwächer ausgeprägten Kompetenzen seltener das Abitur, zehnmal häufiger keinen allgemeinen und dreimal häufiger keinen beruflichen Bildungsabschluss (ebd., S. 38, 92).

Aufgrund der zuwanderungsbedingten Disparitäten muss die Polizei einen Weg finden, die vergleichsweise weniger vorhandenen qualifizierten Migranten mit Bildungsabschluss (mittlerer Schulabschluss oder Abitur) anzusprechen und für den Polizeiberuf zu gewinnen. Die Erfahrungen der Einstellungsbüros zeigen, dass Migranten trotz ihres Bildungsabschlusses wegen mangelnder Sprachkompetenz oft nicht zugelassen werden konnten (Drechsler und Nagora 2007, S. 27 f.). 
4. Zur Notwendigkeit der Einstellung von Migranten: um der Organisation willen oder für eine gelungene Integrationspolitik?

Die Notwendigkeit der vermehrten Einstellung von Migranten in den Polizeivollzugsdienst wird heute nicht mehr in Frage gestellt. Es in allen Bildungs- und Integrationsprogrammen des Bundes und der Länder erklärter Wille, verstärkt Ausländer und Deutsche mit Zuwanderungshintergrund in allen Verwaltungsbereichen des Öffentlichen Dienstes einzustellen, wie es im Nationalen Integrationsplan vorgesehen ist (Die Beauftragte der Bundesregierung für Migration, Flüchtlinge und Integration 2007, S. 17, 28). Ein Indikator für die heutige Akzeptanz sind die Internetpräsenzen der Einstellungsbüros der Polizeien, die auf ein besonderes Interesse an Migranten hinweisen. ${ }^{7}$ Unterschiede gibt es dagegen in den Begründungen der Notwendigkeit, Hunold und Behr unterscheiden die funktionale Begründung (polizeitaktische und personalpolitische Überlegungen) der Organisationspraxis vom integrationspolitischen Ansatz der Forschung (Hunold und Behr 2007, S. 24, dazu auch Bornewasser 2009, S. 37).

Aus polizeitaktischer Sicht sind migrantische Polizeibeamte Milieukenner, die als sprach- und kulturkompetente Insider Zugang zu Migrantencommunities erhalten, um einerseits Ermittlungen in sonst schwer erreichbaren, teils abgeschotteten ethnischen Milieus führen zu können, anderseits das Vertrauen in die Polizei durch ihre augenscheinliche Gleichartigkeit mit der Community zu erhöhen (Behr 2010, S. 11 f., dazu auch Blom 2005, S. 35). Zudem sollen Migranten den durch den demografischen Wandel bedingten Mangel an Personal kompensieren und das Überleben der Organisation sicherstellen (Hunold und Behr 2007, S. 25). Diese Betrachtungsweise kann dazu führen, dass sich Migranten zu Recht auf ihren Nutzen für die Organisation reduziert fühlen, was sich auch in den Ergebnissen dieser Studie zeigte (Majewski 2011, S. 84).

Ein völlig anderes Signal geht dagegen von der Integrationsfunktion aus: gelänge es, die deutsche Polizei oder „das heilige Polizistentum " 8 den in der Gesellschaft repräsentierten Minderheiten entsprechend zu entwickeln, wäre dies von großer Symbolik (Hunold und Behr 2007, S. 23, 25). Dafür wäre allerdings ein neues Selbstverständnis der Organisation nötig, um auf Basis einer Diversity-Politik die Organisationskultur weiterentwickeln zu können (Dittmer 2008, S. 89).

\section{Ursachenforschung}

\subsection{Strukturelle Defizite im Einstellungsverfahren und Barrieren in der Organisation}

Eine Erklärung für die geringen Einstellungszahlen von Migranten ergibt sich aus den üblichen Auswahlverfahren, bei denen sich die Bildungsnachteile von Migranten signifikant niederschlagen.

Die Alternative zur formalen Gleichbehandlung stellt die positive Diskriminierung (affirmative action) von Bewerbern mit $\mathrm{MH}$ dar, wie sie die niederländische Polizei viele Jahre betrieben hat, also eine Besserstellung im Auswahlverfahren durch eine vorab festgelegte Einstellungsquote oder herabgesetzte Anforderungen für Migranten (Hunold und Behr 2007, S. 33; Fijnaut 2010). Niederländische Studien zur affirmative action haben aber ergeben, dass sie dem Ruf allochthoner ${ }^{9}$ Polizisten geschadet hat und diese mitverantwortlich ist für die signifikant hohe Ausstiegsquote dieser Kollegen, die sich massiver polizeiinterner Diskriminierung ausgesetzt sahen (Blom 2005, S. 80). Positive Diskriminierung wird auch deutschen Studien zufolge sowohl von den Polizeibeamten ohne $\mathrm{MH}$, besonders aber von den bevorzugten Migranten abgelehnt (dazu auch Klimke 2010a, S. 34 f. und Zwerg 2008, S. 41).

Kontrovers diskutiert wird, ob und wie sich die Polizei an der Kompensation erkannter Defizite als Resultat einer durch sie nicht zu verantwortenden verfehlten Bildungs- und Integrationspolitik beteiligt. Während sich die Organisation überwiegend für nicht $z u$ ständig erklärt, überwiegt in der Forschung die Forderung, dass das Rekrutierungs- und Integrationsproblem stärker als eigenes Problem anerkannt und nicht mit Hinweis auf die Bildungsdefizite als das der Bewerber betrachtet wird (Holdaway 2010; Hunold 2008, S. 54). In Berlin entschied man sich für eine der Bewerbung vorgeschaltete Möglichkeit der Qualifizierung mittels einer durch die Agentur für Arbeit, den Türkischen Bund Berlin-Brandenburg und den Polizeipräsidenten konzipierten Maßnahme zur besonderen Rekrutierung und Förderung von türkischen Jugendlichen (Hunold 2008, S. 28 und 2010a, S. 211).

Auch andere Hochschulen sind aktiv ${ }^{10}$ : am Albrecht-Dürer-Gymnasium in BerlinNeukölln, an dem nur etwa $15 \%$ der Schü- ler deutsche Eltern haben, werden mehrere Mentorenprojekte (Club Lise ${ }^{11}$ und Teach First ${ }^{12}$ ) durchgeführt, die Jugendliche mit $\mathrm{MH}$ begleiten und für ein Studium bzw. eine Berufsausbildung vorbereiten sollen.

Klimke entdeckte Grenzen der Integrationsfähigkeit durch Widerstand in der Belegschaft (Klimke 2010a, S. 27 f.). Fremde seien sowohl als Sicherheitsrisiko, als auch als Entfremdungsfaktor gefürchtet, da eine spürbare Erhöhung des Migrantenanteils die Mehrheit der Mitglieder und Organisationsstrukturen berührt und zu einem grundlegenden Wandel führen könnte. Folglich wehren sich die strukturkonservativen und modernisierungsskeptischen Mitarbeiter gegen Fremdheit (ebd.). Es bedarf also eines überlegten Change Managements, um Widerstände im Personal zu überwinden.

\subsection{Erfahrungen von Polizeibeamten mit Migrationshintergrund}

Befragt man eingestellte Polizeibeamte mit MH zum Anwerbevorgang, stellen sich polizeiliche Anwerbemaßnahmen als bemerkenswert irrelevant dar: in der Studie von Zwerg waren es in fast allen Fällen persönliche Kontakte zur Polizei bzw. Polizeibeamten, teilweise auch mit $\mathrm{MH}$, die zur Bewerbung führten (Zwerg, S. 34, 67). Hunold stellte in ihrer Studie fest, dass die meisten Studienteilnehmer nicht zielgerichtet den Polizeiberuf anstrebten, sondern eher zufällig und die ersten Anregungen aus dem unmittelbaren sozialen Umfeld kamen; am häufigsten vermittelten bei der Polizei tätige Verwandte, Freunde oder Bekannte Informationen über den Polizeiberuf (Hunold 2010b, S. 71). Keine Rolle spielten dagegen Einstellungsberater oder Mittel der passiven Medien.

Befragt zu Reaktionen auf die Berufswahl im privaten Umfeld wurde überdurchschnittlich oft der elterliche Stolz über den Zugang zum beamteten Staatsdienst genannt, offenbar als manifestierte Bestätigung der Integrationsleistung (Zwerg 2008, S. 43).

\subsection{Mögliche Bewerbungsbarrieren für Interessenten}

Eine der wenigen empirischen Untersuchungen zu Bewerbungsbarrieren stellt die vom englischen Innenministerium in Auftrag gegebenen Studie zu Einstellungen ethnischer Minderheiten gegenüber dem Polizeiberuf als mögliche Karriere dar (Stone and Tuffin 2000). Dort spielten bei den 
Studienteilnehmern vor allen Ängste vor Rassismus in der Polizei eine entmutigende Rolle (Stone and Tuffin 2000, p. VI).

Auch religiöse Vorbehalte wurden identifiziert sowie ein Informationsdefizit bezüglich der Arbeit eines Polizeibeamten (Stone and Tuffin 2000, pp. V - VII), das auch in Deutschland ein Problem darstellen könnte (Dittmer 2008, S. 36).

Ein weiterer Hinderungsgrund kann im Widerstand aus dem Familienkreis bestehen. Hunold stieß auf eine ablehnende Haltung der Eltern, die sie auf die starke Aufstiegsorientierung zurückführte. Eltern übertragen ihre Hoffnungen auf sozialen Aufstieg auf ihre Kinder, wobei dabei weniger die wirtschaftliche Sicherheit und mehr das gesellschaftliche Ansehen eine Rolle spielt (Hunold 2010b, S. 74). Die in Deutschland prestigereichen Berufe wie Arzt ${ }^{13}$ oder Jurist könnten auch in den Herkunftsländern größeres Prestige haben als der des Polizisten.

\section{Erfahrungen europäischer Nachbarn}

Im Vereinigten Königreich ${ }^{14}$ als klassischem Einwanderland führten rassistisch motivierte Ereignisse in den Jahren $1981^{15}$ und $1993^{16}$ zur Einführung eines Rekrutierungsziels durch das Innenministerium: innerhalb von 10 Jahren sollte ein Migrantenanteil von $7 \%$ in den Polizeibehörden erreicht werden (Karakus 2010, S. 225). Dieses Ziel wurde zwar verfehlt, der Anteil von ethnischen Minderheiten an der Gesamtpolizeistärke Englands und Wales' konnte aber immerhin von 2,2\% im Jahr 2000 auf $4,4 \%$ im Jahr 2009, bei der Londoner Metropolitan Police sogar auf 8,8 \% gesteigert werden ${ }^{17}$, Diversität ist nunmehr ein fester Bestandteil der englischen Polizei (Smith 2008; zit. n. Klimke 2010b, S. 180). ${ }^{18}$

In den Niederlanden fördert die Regierung seit 1985 als Teil eines - auch Unternehmen verpflichtenden - Gesamtkonzepts eine aktive durch Monitoring beobachtete Anwerbung von Migranten (Hunold und Behr 2007, S. 33). Im Vordergrund standen dabei jahrelang der Spiegelbildansatz und das integrationspolitische Ziel, wobei die Veränderung und Weiterentwicklung der Organisationskultur in der Polizei betont wurde (Blom 2005, S. 40). Die konsequente quotenregulierte Besserstellung von Migranten im Einstellungsverfahren führte zu massiven Problemen im Personalkörper bis hin zu offener Diskriminierung und in deren Folge zu hohen Abbruchquoten
(Blom 2005, S. 22 ff.). Das Ziel, bis 1995 einen Anteil von $10 \%$ an Migranten im Personal zu haben, wurde verfehlt, tatsächlich wurden 4,8\% erreicht (Hunold und Behr 2007, S. 34). Daraufhin ersetzte man die gescheiterte affirmative action durch zahlreiche spezielle Rekrutierungsmaßnahmen wie Trainee-Programme vor Bewerbungen und Trainingsangebote nach gescheiterten Bewerbungen, um sich später erneut bewerben zu können. Der Anteil von Migranten in der niederländischen Polizei betrug im Jahr $200810 \%$, in Großstädten wie Amsterdam $14 \%$ (Klimke 2010b, S. 180).

Völlig anders stellt sich die Situation in Frankreich dar: nach französischen Selbstverständnis hat jeder Franzose und folglich auch eingebürgerte Migrant $^{19}$ das Recht auf uneingeschränkte Teilnahme am politischen Leben der Nation (Tiemann 2004, S. 25). Aus dem Leitbild der französischen Revolution (Freiheit, Gleichheit, Brüderlichkeit) ergibt sich zum einen, dass von jedem Franzosen eine Anpassung an französische Wert und Kulturmuster, mithin Assimilation erwartet wird, zum anderen, dass keine weitere sozial-demografische Differenzierung zulässig ist, etwa nach Ethnie oder Religion (Klimke 2010, S. 179). Migranten werden folglich nicht gefördert oder gar bevorzugt.

\section{Forschungsfrage und Vorgehensweise}

In Anlehnung an Behr, der zugespitzt formulierte: „Diejenigen, die wollen, können nicht und diejenigen, die können, wollen nicht“ (Behr 2009, S. 153), wurde in dieser Studie der Frage nachgegangen „Warum wollen diejenigen, die können, nicht? “.

Angesichts des explorativen Forschungsvorhabens wurde auf eine qualitative Methode zurückgegriffen und in themenzentrierten Leitfadeninterviews zehn angehende leistungsstarke Abiturienten mit $\mathrm{MH}$ und zwei Schüler ohne MH als Vergleich zu Einstellungen und Motiven bezüglich ihrer Berufswahl und dem Beruf des Polizeibeamten befragt. Es wurde die Fallgruppe der türkischstämmigen Abiturienten gewählt, da Türken die größte ethnische Minderheit in Berlin darstellen (Amt für Statistik Berlin- Brandenburg 2010, S. 12) und sie daher im Sinne des Spiegelbildansatzes die größte Relevanz für Rekrutierungsbemühungen entfalten.

Bei der inhaltlichen Vorbereitung der Interviews wurden die Ansätze der Grounded Theory (Strauss and Corbin 1996) zu Grun- de gelegt, jedoch nicht kategorisch verfolgt, weil auf Vorannahmen nicht verzichtet werden konnte. Um diese ansatzweise empirisch zu stützen, wurden vor Durchführung der Interviews zwei explorative Gespräche mit kürzlich eingestellten, sich derzeit noch im Studium befindlichen Kommissarsanwärtern im 2. Semester an der HWR ${ }^{20}$ geführt.

Der Verfasser wandte sich an Schulleiter dreier Oberschulen in Berlin-Nord-Neukölln ${ }^{21}$ und bat um Vermittlung von türkisch- und deutschstämmigen Abiturienten aus der 13. Klasse, die voraussichtlich ein Abitur mit der Note 2 und besser ablegen werden und sprachkompetent sind, von denen also angenommen werden kann, dass sie erfolgversprechende Bewerber im polizeilichen Auswahlverfahren wären.

Die Auswertung der Daten wurde auf Grundlage eines ständigen Austauschs zwischen den Vorannahmen und den erhobenen Daten im Sinne der Grounded Theory durchgeführt. Herausgearbeitet wurden wiederkehrende Deutungsmuster, die in - auf dem Interviewleitfaden basierenden - Codierungen unter Zuhilfenahme der Software MAXQDA gegliedert und in Auswertungskategorien überführt wurden. Die wesentlichen Aussagemuster wurden unter Einbeziehung exemplarischer Zitate als Ergebnis zusammengefasst.

Den Studienergebnissen vorangestellt wurde statistisches Material zur Entwicklung der Schulabsolventen- und Bewerberzahlen in Berlin sowie eine Darstellung der bisherigen Maßnahmen der Berliner Polizei.

\section{Darstellung der Ergebnisse}

\subsection{Einstellungen zur Berufswahl}

Hinsichtlich der Berufswünsche zeigte sich ein breites Bild mit neun unterschiedlichen Berufsfeldern bei zwölf Befragten. Signifikant war, dass die Studierquote für diese Gruppe bei $100 \%$ lag und damit weit über der durchschnittlichen Studierquote ${ }^{22}$. Der Öffentliche Dienst spielte in den Überlegungen zur Berufswahl keine Rolle.

Befragt nach Faktoren für die Berufswahlentscheidung gaben elf von zwölf Befragten die Arbeitsplatzsicherheit bei einem geregelten Einkommen als wichtiges Berufswahlkriterium an. Nur bei der Hälfte der Befragten spielten Prestigegründe eine Rolle bei der Berufswahl. Zudem sind die prestigereichen Berufe nicht mehr nur auf die des Arztes und Juristen beschränkt, was die Be- 
nennungen anderer Berufe - darunter auch der des Polizeibeamten - belegten.

\subsection{Verständnis vom Polizeiberuf}

Das Wissen um die Aufgaben und Tätigkeiten eines Polizeibeamten war mehrheitlich eher oberflächlich vorhanden und ließ nur vage Vorstellungen von Aufgaben, Tätigkeiten und Strukturen der Polizei erkennen, was angesichts ihrer umfassenden - auch medialen - Präsenz überraschend erscheint. Hier war ein Unterschied zwischen den Abiturienten mit und ohne MH festzustellen, letztere hatten deutlichere Vorstellungen von der Polizei.

Befragt zu Vorteilen des Polizeiberufes stand an der Spitze der Antworten aller Befragten der Beamtenstatus vor dem Respekt, den man erhält. Das Image des Beamtenstatus' war im vollen Umfang präsent, sei es, dass es von den Eltern übertragen oder durch gesellschaftliche Wahrnehmung adoptiert wurde. Im Respekts- bzw. Ansehensgewinn als Polizist und dem Beamtenstatus sind demnach Zugfaktoren für eine mögliche Bewerbung zu sehen.

Als Nachteil des Polizeiberufes wurde am meisten die Gefährlichkeit des Berufes vor den ungünstigen Arbeitszeiten genannt.

Das Ansehen der Berliner Polizei war unter den zwölf befragten Schülern mehrheitlich positiv. So schrieben acht der Befragten der Berliner Polizei ein insgesamt gutes Image $\mathrm{zu}$, wobei in den Bewertungen durchaus differenziert und offen Kritik geäußert wurde. Es wurde dabei auch deutlich zwischen dem Ansehen der Polizei in Berlin und der türkischen Polizei unterschieden und keinesfalls kulturelle Stereotype importiert und übertragen. Bei den von den Befragten ${ }^{23}$ genannten Zuschreibungen für die türkische Polizei lag korrupt vor gewalttätig.

Frauen in der Polizei wurde von allen Befragten grundsätzlich befürwortet, sei es aus Gründen der Gleichberechtigung oder wegen eines besonderen Einfühlungsvermögen und anderen Fähigkeiten, über die Frauen verfügen und mit in den Beruf einbringen. Einige überwiegend männliche türkischstämmige Migranten vertraten jedoch die Meinung, dass Frauen nur eingeschränkt für den der Polizeiberuf geeignet sind, was sich sowohl auf die Motivation möglicher Bewerberinnen auswirken, als auch zu Bewerbungsbarrieren in traditionell patriarchalisch geprägten Familien führen kann.

\subsection{Einstellung zum Polizeiberuf als möglichen eigenen Beruf}

Keiner der Befragten konnte sich zum Zeitpunkt des Interviews vorstellen, in den Polizeivollzugsdienst einzutreten. Die Gründe hierfür waren vielschichtig; am häufigsten wurde die Gefährlichkeit des Berufes als Grund genannt vor den ungünstigen Arbeitszeiten/der schlechten Vereinbarkeit von Familie und Beruf, diese beiden Gründe stellen die größten Bewerbungsbarrieren dar. ${ }^{24}$ Auffällig war, dass acht Befragte vorübergehend den Wunsch hatten, Polizeibeamter zu werden; drei Befragte hatten zudem ein Betriebspraktikum bei der Berliner Polizei absolviert. ${ }^{25}$

Die Annahme der deutschen Staatsangehörigkeit zeigte sich angesichts einer hohen Einbürgerungsquote bei türkischstämmigen Deutschen als nicht relevant, oft unbekannt waren aber die Zulassungsbedingungen im Zusammenhang mit der Staatsangehörigkeit und die Relevanz von vorangegangenen Straffälligkeiten durch nahe Verwandte.

Anders als in England gab es unter den Befragten keine Ängste, für den Beruf Teile ihres kulturellen Hintergrundes aufgeben zu müssen. ${ }^{26}$ Die durch die Studienteilnehmer formulierten Bewerbungsbarrieren erscheinen überwindbar und beruhen teilweise auf dem Medienbild des Polizeiberufes. ${ }^{27}$ Ein Vorteil stellt das grundsätzlich vorhandene breite Interesse am Polizeiberuf dar, an das angeknüpft werden kann, um Interessenten bis zur endgültigen Berufsentscheidung an die Polizei zu binden.

Die Eltern von türkischstämmigen Abiturienten befürworten die Wahl des Polizeiberufes eher, als dass sie sie ablehnen. Selbst wenn Sie die Berufswahl nicht befürworten, respektieren sie den Berufswunsch ihres Kindes. Auch durch den Freundeskreis erfahren sie eher Unterstützung als Ablehnung.

Bei den Befragten überwogen die positiven Erfahrungen mit der Berliner Polizei. Fast alle Befragten gingen jedoch von fremdenfeindlichen Einstellungen unter den Berliner Polizeibeamten im eher geringen Umfang aus, die (noch) kein Entscheidungskriterium gegen eine Bewerbung darstellten. ${ }^{28}$

\subsection{Wahrnehmung von Werbung und des Interesses an Migranten sei- tens der Polizei}

Abiturienten nahmen zwar überwiegend passive Werbemittel und Veranstaltungen an der Schule wahr, rieten aber zu persönlichen Kontaktaufnahmen durch Polizeibeamte mit $\mathrm{MH}$, die die möglichen Interessenten an jugendtypischen Örtlichkeiten mit hohen Migrantenanteil proaktiv aufsuchen. Die meisten Abiturienten mit $\mathrm{MH}$ wussten und befürworteten uneingeschränkt, dass die Polizei verstärkt Migranten als Nachwuchs sucht. Die am häufigsten genannten Gründe dafür waren die Chance zur Integration und der Spiegelbildansatz: ${ }^{29}$

„Ich finde es gut, weil sie [die Polizisten] sehen, wie die Gesellschaft aussieht, die Gesichter der Gesellschaft [...] Die Gesichter der Gesellschaft sind vielfältig, multikulturell und ich denke, so sollte dann halt auch die Polizei aussehen. Kunterbunt“ (Majewski 2011, S. 83).

Bemerkenswert waren in diesem Zusammenhang die Einschränkungen mehrerer Befragter, die Zweifel an der Ehrlichkeit der Bemühungen formulierten:

„Es kann nicht sein, dass man so etwas über eine Quote regelt und sagt, ein bestimmter Anteil muss ausländisch sein. Ich finde das auch keine Lösung, das ist sozusagen etwas Scheinheiliges [...] Es ist diese Vorstellung, die ich insgesamt habe, dass man versucht, Ausländer zu integrieren, aber es eigentlich gar nicht so richtig möchte" (ebd., S. 84).

Die formulierten Zweifel könnten auf die Betonung der organisationsfunktionalen Begründung für das Einstellen von Migranten seitens der Polizei zurückzuführen sei, während die Befragten integrationspolitische Gründe in den Vordergrund stellten.

Bei den Antworten zur Frage, wie die Polizei attraktiver für Migranten werden könnte, dominierte folgerichtig der Wunsch nach einer Willkommenskultur und authentischen Offenheit der Polizei gegenüber Migranten:

„Dass sie halt willkommen sind, dass die Polizei eigentlich darauf wartet, dass sie sich nicht scheuen sollen, sich zu bewerben, dass sie eine Chance bekommen, wenn sie es nur wollen. Dann halt auch sagen, dass sie Vermittler zwischen den ausländischen Bürger und der deutschen Polizei sind, zeigen, dass sie wichtig sind“ (ebd., S. 85).

\section{Fazit}

In der Studie konnten zum einen „harte Faktoren “ identifiziert werden, die dazu führen, dass sich so wenige qualifizierte Migranten bei der Polizei bewerben. Zu diesen zählt, dass die Möglichkeit eines bereits vergüteten Studiums in der Öffentlichkeit genauso wenig bekannt scheint, wie die Vielfältigkeit des Polizeiberufes, der unterschiedliche Interessen bedient und verschiedene Fähigkeiten fordert. Zudem besteht eine Diskrepanz zwischen dem Medienbild des Polizeiberufes und den objektiven Arbeitsbedingungen, die zu großen Bewerbungsbarrieren führt. 
Auch wurde gezeigt, wie wenig die üblichen Werbemaßnahmen geeignet sind, um den Anteil von Migranten in den Polizeien zu steigern. Der Erfolg bei der Rekrutierung von diesen jungen Menschen ist aber nicht nur davon abhängig, welche Arten von Werbemaßnahmen wo platziert werden, sondern auch eine Frage von weichen Faktoren, zu allererst eine Frage der Authenzität. Um als Arbeitgeber für Migranten attraktiver zu werden, muss die Polizei offenbar vor allen glaubwürdiger vermitteln, dass sie sich aus Überzeugung für Menschen mit Migrationshintergrund öffnet und das Ziel verfolgt, vielfältiger zu werden. ${ }^{30}$

Die Ergebnisse dieser Untersuchung zeigen, dass es keinesfalls aussichtslos ist, die Bewerberzahl von geeigneten Migranten zu erhöhen, vorausgesetzt die Polizeien erkennen die Aufgabe als solche als ureigensten Auftrag an und gehen aktiv auf Schulabsolventen zu, um den Faktor Zufall im Berufswahlprozess durch planvolles Handeln zu ersetzen.

Kriminalrat Andreas Majewski M. A. leitet die 1. Kriminalinspektion Verbrechensbekämpfung im Norden Berlins und ist seit mehr als 18 Jahren Kriminalbeamter in Berlin. 2011 graduierte er an der Deutschen Hochschule der Polizei in Münster im Masterstudiengang „Öffentliche Verwaltung - Polizeimanagement". Der Artikel stellt eine Studie vor, die als Masterarbeit an der Deutschen Hochschule der Polizei vorgelegt wurde und 2011 als Buch im Verlag für Polizeiwissenschaften erschienen ist.

\section{Fußnoten}

1 Der zentrale Festakt fand unter Beteiligung von Bundeskanzlerin Angela Merkel und Premierminister Recep Erdogan in Berlin statt $(\mathrm{Zu}-$ griff am 03. Dezember 2011 unter http://www. bundesregierung.de/nn_1264/Content/DE/ Artikel/2011/11/2011-11-02-anwerbeabkommen.html).

$2 \mathrm{Zu}$ Grunde gelegt wird die Definition des Statistischen Bundesamtes: alle nach 1949 auf das heutige Gebiet der Bundesrepublik Deutschland Zugewanderten sowie alle in Deutschland geborenen Ausländer und alle in Deutschland als Deutsche Geborenen mit zumindest einem zugewanderten oder als Ausländer in Deutschland geborenen Elternteil (Statistisches Bundesamt 2007, S. 31).
3 Die Berliner Polizei stellt Deutsche im Sinne des Artikels 116 des Grundgesetzes oder Staatsangehörige eines Mitgliedslandes der Europäischen Union ein, für Angehörige anderer $\mathrm{Na}$ tionalitäten gelten Sonderregelungen (Zugriff am 24. Januar 2011 unter http://www.berlin. de/ polizei/beruf/ausbildung/suk.html\#einstell).

4 Bezüge für den ledigen Anwärter im 1. Jahr: $896,82 €$ brutto/Monat (Zugriff am 21. Mai 2011 unter http://www.berlin.de/imperia/md/ content/polizei/wir-ueberuns/zse/berufsinfos/ verdienstbeispiel_gd_februar_2011.pdf?down load.html).

5 In Berlin beträgt der Anteil von Menschen mit MH bei den unter 6-Jährigen $43 \%$ (Amt für Statistik Berlin-Brandenburg 2011, S. 21, 32).

6 Der Migrationshintergrund wird hier aufgrund der Datenlage für den Bildungsverlauf in Kita, Schulen und Berufsbildung (Kinder- und Jugendhilfestatistik) anders gefasst: ein $\mathrm{MH}$ liegt vor, wenn mindestens ein Elternteil im Ausland geboren wurde (Autorengruppe Bildungsberichterstattung 2010, S. 53).

7 Exemplarisch die Länder NRW, Zugriff am 12. Mai 2011 unter http://www.polizei.nrw. de/ beruf/ihr-weg-zu-uns/voraussetzungen/article/die-grundvoraussetzungen.html, Berlin, Zugriff am 12. Mai 2011 unter http://www. berlin.de/polizei/beruf/berufsinformation.html, Hessen, Zugriff am 12. Mai 2011 unter http:// www.polizei.hessen.de/internetzentral/nav/52d/ 52d40527-bab6-4021-3104-182109241c24. htm sowie Hamburg, Zugriff am 12. Mai 2011 unter http://www.hamburg.de/faq-np/.

8 So einer der in der Studie befragten türkischstämmigen Abiturienten (Majewski 2011, S. 82).

9 Ein von Blom geprägter Begriff für Menschen mit MH (Blom 2005). Altgriechisch für fremd, auswärtig (Quelle: Wikipedia, Zugriff am 12. Mai 2011 unter: http://de.wikipedia.org/ wiki/ Allochthon).

10 Der Tagesspiegel vom 1.März 2011 im Artikel: „Starthilfe für Überflieger. Wie sich Gymnasiasten aus Neuköllner Einwandererfamilien auf Studium und Beruf vorbereiten".

11 Mentorenprojekt der Humboldt-Universität, das sich an naturwissenschaftlich interessierte Schülerinnen - vor allem mit $\mathrm{MH}$ - richtet $(\mathrm{Zu}-$ griff am 12. Mai 2011 unter http://www.clublise.de).

12 Bildungsinitiative, die herausragende Hochschulabsolventen aller Disziplinen an Schulen in sozialen Brennpunkten vermittelt, um benachteiligte Schüler individuell $\mathrm{zu}$ fördern (Zugriff am 12. Mai 2011 unter http://www. teachfirst.de).

13 Nach der Allensbacher Berufsprestige-Skala 2011: 1. Platz Arzt, 7. Platz Rechtsanwalt, 10. Platz: Polizist, Zugriff am 16. Mai 2011 unter: http://www.ifd-allensbach.de/.

14 England, Schottland, Wales und Nordirland.

$15 \mathrm{Im}$ Londoner Stadtteil Brixton wurden vier Tage lang Straßenkontrollen durchgeführt und vor allem schwarze Personen verdachtsunabhängig angehalten und durchsucht. Infolgedessen kam es zu spontanen und zunehmend eskalierenden Ausschreitungen gegenüber der Polizei. Der die Ereignisse untersuchende Richter Scarman stellte fest, dass sich die schwarze Gemeinde von der Polizei erniedrigt und schi- kaniert fühlte und zu wenig Vertrauen in die Polizeiarbeit hatte (Scarman 1981).

16 Im Londoner Stadtteil Eltham wurde der jamaikanische Student Stephen Lawrence von einer Gruppe weißer Jugendlicher erstochen. Der die Ermittlungen untersuchende Richter Macpherson kam zum Schluss, dass die Tat eindeutig rassistisch motiviert war und die Ermittlungen fehlerhaft, schlampig und ihrerseits rassistisch dominiert waren (Macpherson 1999).

17 Das Datenmaterial zu den polizeilichen Personalkörpern bezieht sich auf England und Wales.

18 Beispielhaft kann dafür die Gründung und Institutionalisierung von Minderheitenvertretungen in der Londoner Polizei herangezogen werden (Karakus 2010, S. 226) oder die dienstlich zugelassenen Turbane der indischstämmigen Sikhs unter den Londoner Polizeibeamten im Heise-Online-Artikel „Metropolitan Police räumt mit alten Hüten auf“, Zugriff am 3. Juni 2011 unter http://www.heise.de/tp/arti$\mathrm{kel} / 7 / 7716 / 1 . \mathrm{html}$.

19 In Frankreich geborene Kinder von ausländischen Eltern werden mit Vollendung des 18. Lebensjahres automatisch französische Staatsbürger.

20 Hochschule für Wirtschaft und Recht Berlin. Hier findet die Ausbildung im gehobenen Polizeivollzugsdienst der Polizei Berlin im Rahmen eines Bachelor-Studiums statt.

21 Der Abschnitt liegt in Nord-West Neukölln, der Ausländeranteil an der Wohnbevölkerung beträgt $39 \%$ ohne Deutsche mit $\mathrm{MH}$ (Hoven 2007, S. VII).

22 Der Prognosewert für die Studierquote 2008 lag für Hochschulberechtigte mit MH bei 71$79 \%$ (Autorengruppe Bildungsberichterstattung 2010, S. 289).

23 Dazu gefragt wurden die zehn türkischstämmigen Abiturienten und die zwei Kommissarsanwärter.

24 Weitere Gründe waren u. a. eigene körperliche Ungeeignetheit, sich lebenslang an einen Beruf binden zu müssen oder mögliche Gewissenskonflikte (Majewski 2011, S. 70 ff.).

25 Hier stellt sich die Frage der Qualität und Passung der Praktika zum Praktikanten und sich daraus ergebende Bindungs- und Abschreckeffekten.

26 Stone and Tuffin 2000, p. VI.

27 Dieses spiegelt eben nicht die Bemühungen der Polizei wieder, Familie und Beruf durch flexible Arbeitszeitmodelle vereinbaren zu können und objektiviert auch nicht die tatsächliche Gefährlichkeit des Polizeiberufes (Majewski 2011, S. 73).

28 Die Antworten zu Erfahrungen mit der Polizei und Fremdenfeindlichkeit in Polizei und Gesellschaft werden im Buch in zwei Unterkapiteln ausführlicher dargestellt (Majewski 2011, S. $78 \mathrm{ff}$.)

29 Weitere Gründe waren die besonderen Sprachkompetenzen von Migranten vor einer größeren Akzeptanz der Polizei und dem Abbau von Vorurteilen sowie für ein besseres Verständnis von Migranten (Majewski 2011, S. 83).

30 Dieser Wunsch nach Offenheit und Authenzität steht im Einklang mit der Forderung einer Diversity-Politik in der Forschung (Majewski 2011, S. 14). 